\title{
Volume monogamy of quantum steering ellipsoids for multi-qubit systems
}

\author{
Shuming Cheng, ${ }^{1,2}$ Antony Milne, ${ }^{3}$ Michael J. W. Hall, ${ }^{1}$ and Howard M. Wiseman ${ }^{1}$ \\ ${ }^{1}$ Centre for Quantum Computation and Communication Technology (Australian Research Council), \\ Centre for Quantum Dynamics, Griffith University, Brisbane, QLD 4111, Australia \\ ${ }^{2}$ Key Laboratory of Systems and Control, Academy of Mathematics and Systems Science, \\ Chinese Academy of Sciences, Beijing 100190, P. R. China \\ ${ }^{3}$ Controlled Quantum Dynamics Theory, Department of Physics, \\ Imperial College London, London $S W^{\gamma}$ 2AZ, UK
}

\begin{abstract}
The quantum steering ellipsoid can be used to visualise two-qubit states, and thus provides a generalisation of the Bloch picture for the single qubit. Recently, a monogamy relation for the volumes of steering ellipsoids has been derived for pure 3-qubit states and shown to be stronger than the celebrated Coffman-Kundu-Wootters (CKW) inequality. We first demonstrate the close connection between this volume monogamy relation and the classification of pure 3-qubit states under stochastic local operations and classical communication (SLOCC). We then show that this monogamy relation does not hold for general mixed 3-qubit states and derive a weaker monogamy relation that does hold for such states. We also prove a volume monogamy relation for pure 4-qubit states (further conjectured to hold for the mixed case), and generalize our 3-qubit inequality to $n$ qubits. Finally, we study the effect of noise on the quantum steering ellipsoid and find that the volume of any two-qubit state is non-increasing when the state is exposed to arbitrary local noise. This implies that any volume monogamy relation for a given class of multiqubit states remains valid under the addition of local noise. We investigate this quantitatively for the experimentally relevant example of isotropic noise.
\end{abstract}

\section{INTRODUCTION}

Qubits play a fundamental role in quantum information processing tasks [1] and quantum measurement and control [2]. The Bloch vector faithfully captures all features of a single qubit state. However, finding an analogous geometric representation of multi-qubit states is harder. An elegant solution to this problem has recently been given for the two-qubit case [3]. In particular, for a two-qubit state shared between two parties, Alice and Bob say, the set of all possible Bloch vectors that Alice can steer Bob's qubit to, via all possible local measurements on her qubit, forms an ellipsoid, called the quantum steering ellipsoid. Together with Alice's and Bob's local Bloch vectors, the quantum steering ellipsoid provides a geometric representation of the shared 2-qubit state [3].

The set of two-qubit states has a rich structure, which is mirrored, and in some cases added to, by a corresponding zoo of quantum steering ellipsoids $\underline{3}$. For example, properties of steering ellipsoids reflect various features of quantum correlations, such as discord [3 5], entanglement [3, 6, 7] and Einstein-Podolsky-Rosen (EPR) steering 8 11. Moreover, it appears that steering ellipsoids may be useful for characterising correlation properties of multiqubit states, such as monogamy, in new ways. In particular, it is well known that quantum correlations cannot be freely shared between members of multipartite systems, resulting in monogamy relations for, e.g., concurrence [12, 13, Bell nonlocality [14-17, and EPRsteering inequalities [18]. In this vein, Milne et al. have recently obtained a strong monogamy relation for the volumes of the steering ellipsoids generated by pure 3-qubit states, termed volume monogamy [6], which is strictly stronger than the well known Coffman-Kundu-Wootters (CKW) monogamy relation for concurrence 12 in the pure regime.

The volume monogamy relation for pure 3-qubit states immediately suggests a number of questions: Does it discriminate between different types of entanglement? Is it valid for the mixed case? Are there similar relations for multiqubit states? And what happens when noise, induced by inevitable interaction with the environment and imperfections of local measurements, is present? We will provide some answers to these questions in this paper, following a brief review of the quantum steering ellipsoid and its properties in Sec. III

In Sec. III we obtain and discuss volume monogamy relations in general scenarios ranging from pure 3-qubit states to general multiqubit states. First, in Sec. III A we discuss the known volume monogamy relation for pure 3-qubit states [6]; give an alternative proof of this relation that demonstrates a link with the quantum marginal problem [19; review the derivation of the CKW monogamy relation from volume monogamy; and establish a close connection between properties of volume monogamy and the classification of pure 3-qubit states under stochastic local operations and classical communication (SLOCC). In Sec. III B we show that this volume monogamy relation is violated by some mixed states, and derive a weaker volume monogamy relation that is valid for all 3-qubit states, pure or mixed. We obtain a monogamy relation of the same form for pure 4-qubit states in Sec. III C, which we conjecture is also applicable to mixed states, and give a nontrivial volume monogamy relation for general multiqubit states in Sec. III D.

In Sec. IV we investigate the effects of noise on steering ellipsoid volumes and on volume monogamy relations. In 
Sec. IV A we show that the volume of the steering ellipsoid decreases monotonically under arbitrary local operations, including under local noise channels in particular. This significantly generalises a recent result [20, which is restricted to particular classes of states and types of noise. Moreover, it implies that any volume monogamy relation for a given set of multiqubit states remains valid under the addition of local noise. This includes, in particular, the strong volume monogamy relation for pure 3 -qubit states in Ref. 6. Finally, in Sec. IV B we investigate the experimentally relevant case of isotropic noise acting on a family of 3-qubit W-class states.

We conclude with some remarks and open questions in Sec. V]

\section{QUANTUM STEERING ELLIPSOIDS FOR TWO-QUBIT STATES}

Any two-qubit state $\rho_{A B}$ can be written in the form

$$
\begin{gathered}
\rho_{A B}=\frac{1}{4}\left(\mathbb{1}_{A} \otimes \mathbb{1}_{B}+\mathbf{a} \cdot \boldsymbol{\sigma} \otimes \mathbb{1}_{B}+\mathbb{1}_{A} \otimes \mathbf{b} \cdot \boldsymbol{\sigma}\right. \\
\left.+\sum_{j, k=1}^{3} T_{j k} \sigma_{j} \otimes \sigma_{k}\right),
\end{gathered}
$$

where $\boldsymbol{\sigma} \equiv\left(\sigma_{1}, \sigma_{2}, \sigma_{3}\right)$ denotes the vector of Pauli spin operators and $\mathbb{1}_{A}, \mathbb{1}_{B}$ are identity operators. Here $\mathbf{a}$ and $\mathbf{b}$ are the Bloch vectors of Alice's and Bob's reduced states and $T$ is the spin correlation matrix, i.e.,

$$
\begin{aligned}
a_{j} & :=\operatorname{Tr}\left[\rho_{A B} \sigma_{j} \otimes \mathbb{1}_{B}\right], \quad b_{k}:=\operatorname{Tr}\left[\rho_{A B} \mathbb{1}_{A} \otimes \sigma_{k}\right], \\
T_{j k} & :=\operatorname{Tr}\left[\rho_{A B} \sigma_{j} \otimes \sigma_{k}\right], \quad(j, k=1,2,3) .
\end{aligned}
$$

Different choices of Alice's local measurements result in different steered states for Bob. Specifically, each local measurement outcome for Alice corresponds to some element $E \geq 0$ of a positive operator-valued measure (POVM) describing her measurement. Thus, $E=e_{0}\left(\mathbb{1}_{A}+\mathbf{e} \cdot \boldsymbol{\sigma}\right)$, with $e_{0} \geq 0$ and $|\mathbf{e}| \leq 1$. This outcome is obtained with probability

$$
p^{E}=\operatorname{Tr}\left[\rho_{A B} E \otimes \mathbb{1}_{B}\right]=e_{0}(1+\mathbf{a} \cdot \mathbf{e}),
$$

leading to the steered state

$$
\rho_{B}^{E}=\frac{\operatorname{Tr}_{A}\left[\rho_{A B} E \otimes \mathbb{1}_{B}\right]}{p^{E}}=\frac{1}{2}\left(\mathbb{1}_{B}+\frac{\left(\mathbf{b}+T^{\top} \mathbf{e}\right) \cdot \boldsymbol{\sigma}}{1+\mathbf{a} \cdot \mathbf{e}}\right)
$$

for Bob's qubit.

Considering all possible local measurements by Alice, it follows that the corresponding set of Bob's steered states is represented by the set of Bloch vectors

$$
\mathcal{E}_{B \mid A}=\left\{\frac{\mathbf{b}+T^{\top} \mathbf{e}}{1+\mathbf{a} \cdot \mathbf{e}}:|\mathbf{e}| \leq 1\right\} .
$$

While not immediately apparent from Eq. (4), this set forms a (possibly degenerate) ellipsoid, and hence is called a quantum steering ellipsoid [3]. The subscript $B \mid A$ indicates the steering ellipsoid for Bob that is generated by Alice's local measurements. Similarly, there is a steering ellipsoid for Alice generated by Bob's local measurements, denoted by $\mathcal{E}_{A \mid B}$. The ellipsoid $\mathcal{E}_{B \mid A}$ is fully determined by its centre,

$$
\mathbf{c}_{B \mid A}=\frac{\mathbf{b}-T^{\top} \mathbf{a}}{1-a^{2}}
$$

and its orientation matrix,

$$
Q_{B \mid A}=\frac{1}{1-a^{2}}\left(T-\mathbf{a b}^{\top}\right)^{\top}\left(I+\frac{\mathbf{a a}^{\top}}{1-a^{2}}\right)\left(T-\mathbf{a b}^{\top}\right),
$$

where the eigenvalues and corresponding eigenvectors of $Q_{B \mid A}$ describe the squared lengths of the ellipsoid's semiaxes and their orientations [3]. Here and elsewhere we use $x$ to denote $|\mathbf{x}|$ for the vector $\mathbf{x}$.

The quantum steering ellipsoid $\mathcal{E}_{B \mid A}$, together with the reduced Bloch vectors $\mathbf{a}$ and $\mathbf{b}$, provides a faithful representation of any two-qubit state up to local unitary operations on Alice's qubit [3]. Additionally, its various geometric properties encode useful information about the state. For example, the state is separable if and only if its steering ellipsoid can be nested in a tetrahedron that is in turn nested in the Bloch sphere [3].

The size of the steering ellipsoid is quantified by its volume [3],

$$
V_{B \mid A}=\frac{4 \pi}{3} \frac{\left|\operatorname{det}\left(T-\mathbf{a b}^{\top}\right)\right|}{\left(1-a^{2}\right)^{2}} .
$$

It is obvious that the steering ellipsoid is constrained to lie inside the Bloch sphere, implying the volume is always no larger than $V_{\text {unit }}=\frac{4 \pi}{3}$. It is therefore convenient to work with the corresponding normalised volume defined by

$$
v_{B \mid A}:=\frac{V_{B \mid A}}{V_{\text {unit }}} \leq 1 .
$$

The upper bound is achieved if and only if Alice and Bob share a pure entangled 2-qubit state [3], and hence the steering ellipsoids of such states coincide with the Bloch ball. In contrast, the normalised volume of any separable state is restricted by the nested tetrahedron condition to be no greater than $1 / 27[3$. Thus, the steering ellipsoid volume is, at least to some extent, connected with the entanglement of the state. This paper will explore this connection further, via volume monogamy relations.

For later reference, we note here that the quantum steering ellipsoid $\mathcal{E}_{B \mid A}$, and hence its volume $V_{B \mid A}$, is invariant under the local filtering operation on Alice's qubit defined by [6]

$$
\tilde{\rho}_{A B}:=\left[\left(2 \rho_{A}\right)^{-1 / 2} \otimes \mathbb{1}_{B}\right] \rho_{A B}\left[\left(2 \rho_{A}\right)^{-1 / 2} \otimes \mathbb{1}_{B}\right] .
$$

The filtered state $\tilde{\rho}_{A B}$ is called the canonical form of $\rho_{A B}$, and has the useful properties $\tilde{\mathcal{E}}_{B \mid A}=\mathcal{E}_{B \mid A}$ and $\tilde{\mathbf{a}}=\mathbf{0}$. 
Thus, for example, the normalised volume of $\mathcal{E}_{B \mid A}$ can be rewritten in the simple form

$$
v_{B \mid A}=\tilde{v}_{B \mid A}=\left|\operatorname{det} \tilde{T}_{A B}\right|,
$$

via Eqs. (5) and (6), where $\tilde{T}_{A B}$ denotes the spin correlation matrix for $\tilde{\tilde{\rho}}_{A B}$. Note that the canonical form is well-defined whenever Alice does not have a pure qubit state, i.e., whenever $a \neq 1$. Conversely, for $a=1$ the shared state factorises, and hence Bob's steering ellipsoid trivially reduces to the single point $\mathcal{E}_{B \mid A}=\{\mathbf{b}\}$, with $v_{B \mid A}=0$.

\section{VOLUME MONOGAMY}

\section{A. Pure 3-qubit states}

\section{Monogamy, quantum marginals and concurrence}

Consider now the scenario in which Alice, Bob, and Charlie share a pure 3-qubit state, $\rho_{A B C}=$ $\left|\psi_{A B C}\right\rangle\left\langle\psi_{A B C}\right|$, and let $\mathcal{E}_{B \mid A}$ and $\mathcal{E}_{C \mid A}$ denote the steering ellipsoids for Bob and Charlie, respectively, generated by local measurements on Alice's qubit. Milne et al. have derived the strong volume monogamy relation [6, 21]

$$
\sqrt{v_{B \mid A}}+\sqrt{v_{C \mid A}} \leq 1
$$

for the corresponding normalised volumes.

This relation immediately implies that Alice cannot steer both Bob and Charlie to a large set of states. For example, if Alice is able to steer Bob to the whole Bloch sphere (i.e., they share a pure entangled state), then Charlie's steering ellipsoid has zero volume (and indeed reduces to a single point). The volume monogamy relation (9) is depicted in Fig. 1. It is nontrivially saturated if and only if $\left|\psi_{A B C}\right\rangle$ is a W-class state [6, 21] (see also Sec. III A.2 below).

We note here an alternative proof of Eq. $(9)$ to that given in Ref. 21, which does not require consideration of extremal ellipsoid volumes, and which provides an interesting connection between volume monogamy and the quantum marginal problem [19, 22. For $a=1$ the proof is trivial: Alice's state is pure and hence the shared state factorises, implying that $v_{B \mid A}=v_{C \mid A}=0$. Otherwise, for $a \neq 1$ we can apply a local filtering operation similarly to Eq. (7), with $\mathbb{1}_{B}$ replaced by $\mathbb{1}_{B C}$ and $\rho_{A B}$ replaced by $\rho_{A B C}$, to obtain the corresponding canonical state $\tilde{\rho}_{A B C}$ with $\tilde{\mathbf{a}}=\mathbf{0}$. Taking partial traces, the normalised volumes of Bob's and Charlie's steering ellipsoids follow via Eq. (8) as

$$
\begin{aligned}
& v_{B \mid A}=\tilde{v}_{B \mid A}=\left|\operatorname{det} \tilde{T}_{A B}\right|=\tilde{c}^{2}, \\
& v_{C \mid A}=\tilde{v}_{C \mid A}=\left|\operatorname{det} \tilde{T}_{A C}\right|=\tilde{b}^{2},
\end{aligned}
$$

respectively, where the final equalities may be proved for pure canonical states by direct calculation, or via partial transposition properties as per Ref. 21. Finally, we

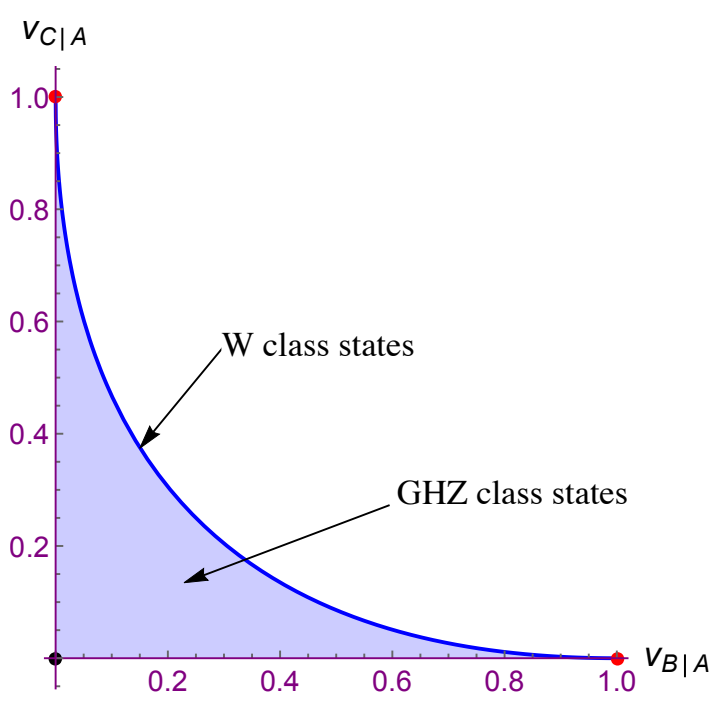

FIG. 1. The volume monogamy relation, Eq. 9, for pure states. The $x$-axis and $y$-axis refer to the normalised volumes $v_{B \mid A}$ and $v_{C \mid A}$ respectively. Both fully factorisable states and bipartite entangled states $\left|\psi_{A}\right\rangle\left|\psi_{B C}\right\rangle$ are mapped to the origin. Two red points $(1,0)$ and $(0,1)$ correspond to the other two classes of bipartite entangled states $\left|\psi_{A B}\right\rangle\left|\psi_{C}\right\rangle$ and $\left|\psi_{A C}\right\rangle\left|\psi_{B}\right\rangle$. The blue solid line is $\sqrt{v_{A \mid B}}+\sqrt{v_{C \mid B}}=1$ and represents the $\mathrm{W}$-class states, except for the red points $(1,0)$ and $(0,1)$. The shaded region, including the two axes, represents the GHZ-class states.

apply the polygon inequality [19, 22.

$$
b+c \leq 1+a,
$$

for the Bloch vectors of any pure 3-qubit state, to the canonical state $\tilde{\rho}_{A B C}$, yielding

$$
\sqrt{v_{B \mid A}}+\sqrt{v_{C \mid A}}=\tilde{c}+\tilde{b} \leq 1+\tilde{a}=1
$$

as required.

Remarkably, the volume monogamy relation (9) also has a deep connection with entanglement monogamy. In particular, the concurrence of a bipartite state $\rho_{A B}$ has the upper bound [6]

$$
C^{2}\left(\rho_{A B}\right) \leq\left(1-a^{2}\right) \sqrt{v_{B \mid A}},
$$

which combined with Eq. (9) immediately yields the celebrated CKW inequality [12],

$$
C^{2}\left(\rho_{A B}\right)+C^{2}\left(\rho_{A C}\right) \leq\left(1-a^{2}\right)=4 \operatorname{det} \rho_{A} .
$$

Thus, volume monogamy is a mathematically stronger condition than the monogamy of concurrence. Note, however, the latter is valid for any 3-qubit state, including mixed states. This is not the case for Eq. (9), as we will show in Sec. III B.

\section{Connection to SLOCC classes and 3-tangle}

It has been shown in Ref. 23 that any pure 3-qubit state can be classified into one of six entanglement 
classes, with all members of any one class being interconvertible under SLOCC transformations, i.e., under local operations and classical communication with some nonzero probability. Here we show that these classes map onto corresponding regions of Fig. 1, thus relating these classes to properties of steering ellipsoid volumes.

We first consider the four classes having no tripartite entanglement. The first of these comprises all fully factorisable states, i.e., those of the form $\left|\psi_{A B C}\right\rangle=$ $\left|\psi_{A}\right\rangle\left|\psi_{B}\right\rangle\left|\psi_{C}\right\rangle$. All steering ellipsoids reduce to single points for this class, with zero volumes, and thus it is mapped to the origin in Fig 1. Next are the three classes of bipartite entangled states, with the corresponding forms $\left|\psi_{A}\right\rangle\left|\psi_{B C}\right\rangle,\left|\psi_{A B}\right\rangle\left|\psi_{C}\right\rangle$ and $\left|\psi_{A C}\right\rangle\left|\psi_{B}\right\rangle$. For the first of these, Alice's qubit is uncorrelated with Bob's and Charlie's qubits, and hence the steering ellipsoids $\mathcal{E}_{B \mid A}$ and $\mathcal{E}_{C \mid A}$ again have zero volumes, corresponding to the origin in Fig. 1. For the other two bipartite classes, Alice can steer one of Bob and Charlie's qubits to the entire Bloch sphere, and the other to a single point. Thus, these two classes correspond to the two red dots in Fig. 1, and trivially saturate volume monogamy relation $(9)$.

The remaining two entanglement classes comprise genuine 3-party entangled states. They correspond to states which are convertible, under SLOCC transformations, to either the W-state

$$
\left|\psi_{A B C}\right\rangle=\frac{1}{\sqrt{3}}(|100\rangle+|010\rangle+|001\rangle),
$$

or to the Greenberger-Horne-Zeilinger (GHZ) state

$$
\left|\psi_{A B C}\right\rangle=\frac{1}{\sqrt{2}}(|000\rangle+|111\rangle),
$$

and are called the $\mathrm{W}$-class and the GHZ-class, respectively 23 . These two classes are distinguished by having 3-tangles $\tau\left(\rho_{A B C}\right)=0$ and $\tau\left(\rho_{A B C}\right)>0$, respectively [23], where for pure states [12]

$$
\tau\left(\rho_{A B C}\right):=4 \operatorname{det} \rho_{A}-C^{2}\left(\rho_{A B}\right)-C^{2}\left(\rho_{A C}\right) .
$$

As indicated in Fig. 1, and demonstrated below, W-class states are precisely those states which nontrivially saturate the volume monogamy relation, while the GHZ-class states correspond to the entire region below this saturating curve.

It has been shown by Milne et al. that the volume monogamy relation is saturated if and only if the state is a 'maximum volume' state, with the canonical form

$$
\left|\tilde{\psi}_{A B C}\right\rangle=(|100\rangle+\cos \theta|010\rangle+\sin \theta|001\rangle) / \sqrt{2}
$$

up to local unitary transformations, where $\theta \in[0, \pi / 2] 6$, 21. It is straightforward to check that such states are bipartite entangled states for $\theta=0, \pi / 2$, corresponding to the points $(0,1)$ and $(1,0)$ in Fig. 1 , and are $\mathrm{W}$-class states otherwise 23. Hence, all states nontrivially saturating the volume monogamy relation are $\mathrm{W}$-class states.
Conversely, noting that $\mathrm{W}$-class states have zero 3-tangle and $a<1$ [23], the inequality

$$
\tau\left(\rho_{A B C}\right) \geq\left(1-a^{2}\right)\left(1-\sqrt{v_{B \mid A}}-\sqrt{v_{C \mid A}}\right)
$$

following from Eqs. (13) and 17) immediately implies that every $\mathrm{W}$-class state saturates the monogamy relation.

It follows from the above that all GHZ-class states must correspond to points in the region below the saturating curve in Fig. 1. We show that indeed every point in this region, including the axes, corresponds to such a state. In particular, we give a family of canonical GHZclass states for which the normalised volumes are mapped to every point $(x, y)$ in the shaded area in Fig. 1, including the axes. The explicit form of these states is defined via two real free parameters:

$$
\begin{aligned}
\left|\tilde{\psi}_{A B C}\right\rangle=\frac{1}{\sqrt{2}} & (\sin \alpha|100\rangle+\sin \beta|010\rangle \\
& +\cos \beta|001\rangle+\cos \alpha|111\rangle)
\end{aligned}
$$

where $\alpha, \beta \in(0, \pi / 2)$, which immediately maps to the coordinates $x=v_{B \mid A}=\frac{1}{4}(\cos 2 \alpha+\cos 2 \beta)^{2}$ and $y=$ $v_{C \mid A}=\frac{1}{4}(\cos 2 \alpha-\cos 2 \beta)^{2}$ via Eqs. 10 and 111 . These fill the shaded area because any point $(x, y)$ therein corresponds to either: $2 \alpha=\arccos (\sqrt{x}+\sqrt{y})$ and $2 \beta=\arccos (\sqrt{x}-\sqrt{y})$; or $2 \alpha=\arccos (\sqrt{x}-\sqrt{y})$ and $2 \beta=\arccos (\sqrt{x}+\sqrt{y})$.

\section{B. Mixed 3-qubit states}

A natural question to consider is whether the volume monogamy relation $(9$ is also valid for a general mixed 3-qubit state. Unfortunately, the answer is negative. A counterexample is given by

$$
\rho_{A B C}=\frac{1}{2}\left(\left|\chi_{1}\right\rangle\left\langle\chi_{1}|+| \chi_{2}\right\rangle\left\langle\chi_{2}\right|\right)
$$

with

$$
\begin{aligned}
& \left|\chi_{1}\right\rangle=\frac{1}{\sqrt{6}}(|101\rangle-2|011\rangle+|110\rangle), \\
& \left|\chi_{2}\right\rangle=\frac{1}{\sqrt{6}}(|010\rangle-2|100\rangle+|001\rangle) .
\end{aligned}
$$

The 2-qubit reduced states $\rho_{A B}$ and $\rho_{A C}$ are then identical Werner states, of the form

$$
\frac{2}{3}\left|\psi^{-}\right\rangle\left\langle\psi^{-}\right|+\frac{1}{3} \frac{\mathbb{1}}{2} \otimes \frac{\mathbb{1}}{2},
$$

where $\left|\psi^{-}\right\rangle$denotes the singlet state $(|01\rangle-|10\rangle) / \sqrt{2}$. It is easy to verify that the corresponding steering ellipsoids are spheres of radius $2 / 3\left[3\right.$. Hence, $\sqrt{v_{B \mid A}}+$ $\sqrt{v_{C \mid A}}=2 \sqrt{8 / 27}=1.0888>1$, implying that the volume monogamy relation $(9)$ does not hold for all mixed 3-qubit states. 
In Sec. IV we will show that Eq. (9) does remain valid for the particular case of mixed states obtained via local operations on pure 3-qubit states. Here, however, we will derive a volume monogamy relation that is valid for all 3-qubit states:

$$
\left(v_{B \mid A}\right)^{2 / 3}+\left(v_{C \mid A}\right)^{2 / 3} \leq 1 .
$$

This monogamy relation is clearly weaker than Eq. (9) for pure states. However, it is saturated in some casesfor example, when $\rho_{A B C}=\rho_{A B} \otimes \rho_{C}$ where $\rho_{A B}$ is a pure entangled state, in which case $v_{B \mid A}=1$ and $v_{C \mid A}=0$.

Our derivation of Eq. 22 is based on a relatively strong tradeoff relation for the pairwise spin correlations of any 3-qubit state $\rho_{A B C}$,

$$
\operatorname{Tr}\left[T_{A B}^{\top} T_{A B}\right]+\operatorname{Tr}\left[T_{A C}^{\top} T_{A C}\right]+\operatorname{Tr}\left[T_{B C}^{\top} T_{B C}\right] \leq 3
$$

(with equality for pure states), obtained as follows. First, consider a pure state $\rho_{A B C}$. From the Schmidt decomposition, the purities of any bipartition of such a state satisfy $\operatorname{Tr}\left[\rho_{A B}^{2}\right]=\operatorname{Tr}\left[\rho_{C}^{2}\right], \operatorname{Tr}\left[\rho_{A C}^{2}\right]=\operatorname{Tr}\left[\rho_{B}^{2}\right]$, and $\operatorname{Tr}\left[\rho_{B C}^{2}\right]=\operatorname{Tr}\left[\rho_{A}^{2}\right]$, which from the definitions in Eq. (2) are equivalent to

$$
\begin{aligned}
& \operatorname{Tr}\left[T_{A B}^{\top} T_{A B}\right]+a^{2}+b^{2}=1+2 c^{2}, \\
& \operatorname{Tr}\left[T_{A C}^{\top} T_{A C}\right]+a^{2}+c^{2}=1+2 b^{2}, \\
& \operatorname{Tr}\left[T_{B C}^{\top} T_{B C}\right]+b^{2}+c^{2}=1+2 a^{2} .
\end{aligned}
$$

Summing these three equations immediately leads to the identity

$$
\operatorname{Tr}\left[T_{A B}^{\top} T_{A B}\right]+\operatorname{Tr}\left[T_{A C}^{\top} T_{A C}\right]+\operatorname{Tr}\left[T_{B C}^{\top} T_{B C}\right]=3
$$

for pure 3-qubit states. Second, expressing a mixed state $\rho_{A B C}$ as a convex combination of pure states, $\rho_{A B C}=$ $\sum_{m} p_{m}\left|\varphi_{m}\right\rangle\left\langle\varphi_{m}\right|$, and letting $T_{A B}^{m}$ denote the spin correlation matrix of $\operatorname{Tr}_{C}\left|\varphi_{m}\right\rangle\left\langle\varphi_{m}\right|$, we have

$$
\begin{aligned}
& \operatorname{Tr}\left[T_{A B}^{\top} T_{A B}\right] \\
& =\sum_{m, n} p_{m} p_{n} \operatorname{Tr}\left[\left(T_{A B}^{m}\right)^{\top} T_{A B}^{n}\right] \\
& \leq \sum_{m, n} p_{m} p_{n}\left(\operatorname{Tr}\left[\left(T_{A B}^{m}\right)^{\top} T_{A B}^{m}\right] \operatorname{Tr}\left[\left(T_{A B}^{n}\right)^{\top} T_{A B}^{n}\right]\right)^{1 / 2} \\
& \leq \frac{1}{2} \sum_{m, n} p_{m} p_{n}\left(\operatorname{Tr}\left[\left(T_{A B}^{m}\right)^{\top} T_{A B}^{m}\right]+\operatorname{Tr}\left[\left(T_{A B}^{n}\right)^{\top} T_{A B}^{n}\right]\right) \\
& =\sum_{m} p_{m} \operatorname{Tr}\left[\left(T_{A B}^{m}\right)^{\top} T_{A B}^{m}\right] .
\end{aligned}
$$

Here the first inequality follows from the Schwarz inequality, and the second from the geometric mean being no greater than the arithmetic mean. One has similar relations for $\operatorname{Tr}\left[T_{A C}^{\top} T_{A C}\right]$ and $\operatorname{Tr}\left[T_{B C}^{\top} T_{B C}\right]$. Summing these and using identity (24) for pure states then yields Eq. 23 as required.

We note that Eq. 23) can itself be considered as a monogamy relation, for the strengths of the pairwise spin correlations, and subsumes the known monogamy relation for pair-wise Bell nonlocality 24.

Similarly to the proof of Eq. (9) in the previous section, we now consider the canonical state $\tilde{\rho}_{A B C}$ for $a \neq 1$ (since Eq. (22) is similarly trivially satisfied when $a=1$ ). Equation (8) then yields

$$
\begin{aligned}
\left(v_{B \mid A}\right)^{2 / 3}+\left(v_{C \mid A}\right)^{2 / 3} & \\
& =\left|\operatorname{det}\left[\left(\tilde{T}_{A B}\right)^{\top} \tilde{T}_{A B}\right]\right|^{1 / 3}+\left|\operatorname{det}\left[\left(\tilde{T}_{A C}\right)^{\top} \tilde{T}_{A C}\right]\right|^{1 / 3} \\
& \leq \frac{1}{3} \operatorname{Tr}\left[\left(\tilde{T}_{A B}\right)^{\top} \tilde{T}_{A B}\right]+\frac{1}{3} \operatorname{Tr}\left[\left(\tilde{T}_{A C}\right)^{\top} \tilde{T}_{A C}\right] \\
& \leq 1
\end{aligned}
$$

as desired. Here the first inequality follows from the arithmetic-geometric mean inequality, applied to the eigenvalues of $\left(\tilde{T}_{A B}\right)^{\top} \tilde{T}_{A B}$ and $\left(\tilde{T}_{A C}\right)^{\top} \tilde{T}_{A C}$, and the second inequality from the tradeoff relation in Eq. (23).

In the next section we show that a monogamy relation of the same form (involving $2 / 3$ powers) also holds for pure 4-qubit states.

\section{4-qubit states}

We first remark that the strong monogamy relation (9) for pure 3-qubit states cannot be generalised to a similar form for pure n-qubit states, for any $n \geq 4$. In particular, by purifying the counterexample in Eq. 20, we can construct the pure 4-qubit state

$$
|\psi\rangle_{A B C D}=\frac{1}{\sqrt{2}}\left(\left|\chi_{1}\right\rangle_{A B C}|0\rangle_{D}+\left|\chi_{2}\right\rangle_{A B C}|1\rangle_{D}\right)
$$

implying, similarly to the counterexample, that $\sqrt{v_{B \mid A}}+$ $\sqrt{v_{C \mid A}}+\sqrt{v_{D \mid A}}=2 \sqrt{8 / 27}+\sqrt{v_{D \mid A}}>1$. It follows more generally, by considering an n-qubit pure state with factor $|\psi\rangle_{A B C D}$, that the form of Eq. (9) does not generalise to any $n \geq 4$.

We will show here, however, that the volume monogamy relation

$$
\left(v_{B \mid A}\right)^{2 / 3}+\left(v_{C \mid A}\right)^{2 / 3}+\left(v_{D \mid A}\right)^{2 / 3} \leq 1
$$

is valid for any pure 4 -qubit state $\rho_{A B C D}$, and give numerical evidence strongly supporting its validity for mixed 4-qubit states.

To prove result (28), we adapt the techniques used in the proof of Eq. 22) in Sec. IIIB. First, using the equalities of purities of any bipartition of a pure state, we have

$$
\begin{aligned}
& a^{2}+b^{2}+\operatorname{Tr}\left[T_{A B}^{\top} T_{A B}\right]=c^{2}+d^{2}+\operatorname{Tr}\left[T_{C D}^{\top} T_{C D}\right], \\
& a^{2}+c^{2}+\operatorname{Tr}\left[T_{A C}^{\top} T_{A C}\right]=b^{2}+d^{2}+\operatorname{Tr}\left[T_{B D}^{\top} T_{B D}\right], \\
& a^{2}+d^{2}+\operatorname{Tr}\left[T_{A D}^{\top} T_{A D}\right]=b^{2}+c^{2}+\operatorname{Tr}\left[T_{B C}^{\top} T_{B C}\right],
\end{aligned}
$$

with respect to the bipartitions $(A B, C D),(A C, B D)$ and $(A D, B C)$. We also have, for the bipartition 
$(A, B C D)$,

$$
\begin{aligned}
b^{2}+c^{2}+d^{2} & +\operatorname{Tr}\left[T_{B C}^{\top} T_{B C}+T_{B D}^{\top} T_{B D}+T_{C D}^{\top} T_{C D}\right] \\
& +L_{B C D}=3+4 a^{2},
\end{aligned}
$$

where

$$
L_{B C D}:=\sum_{l, m, n}\left(\operatorname{Tr}\left[\mathbb{1} \otimes \sigma_{l} \otimes \sigma_{m} \otimes \sigma_{n} \rho_{A B C D}\right]\right)^{2}
$$

is a measure of the tripartite spin correlation strength between Bob, Charlie and Dianne. Summing Eqs. (29)(32) yields

$$
\begin{aligned}
& \operatorname{Tr}\left[\left(T_{A B}^{\top} T_{A B}\right]+\operatorname{Tr}\left[T_{A C}^{\top} T_{A C}\right]+\operatorname{Tr}\left[T_{A D}^{\top} T_{A D}\right]\right. \\
& =3+a^{2}-L_{B C D} \leq 3+a^{2} .
\end{aligned}
$$

Applying a local filtering operation similarly to Eq. (7), with $\mathbb{1}_{B}$ replaced by $\mathbb{1}_{B C D}$ and $\rho_{A B}$ replaced by $\rho_{A B C D}$, to obtain the corresponding canonical state $\tilde{\rho}_{A B C D}$, we have $\tilde{\mathbf{a}}=\mathbf{0}$ and hence that

$\operatorname{Tr}\left[\left(\tilde{T}_{A B}\right)^{\top} \tilde{T}_{A B}\right]+\operatorname{Tr}\left[\left(\tilde{T}_{A C}\right)^{\top} \tilde{T}_{A C}\right]+\operatorname{Tr}\left[\left(\tilde{T}_{A D}\right)^{\top} \tilde{T}_{A D}\right] \leq 3$.

Eq. (28) then follows via the same arguments used in the derivation of Eq. 267.

Finally, we conjecture that inequality $(35)$ generalises to

$$
\operatorname{Tr}\left[T_{A B} T_{A B}^{\top}\right]+\operatorname{Tr}\left[T_{A C} T_{A C}^{\top}\right]+\operatorname{Tr}\left[T_{A D} T_{A D}^{\top}\right] \leq 3 .
$$

for all pure 4-qubit states. We have employed numerical simulations to generate $2 \times 10^{5}$ random pure states and find no violation of inequality 36 . The validity of this conjecture would imply, using the same techniques as above, that monogamy relation (28) in fact holds for all 4-qubit states.

\section{Multiqubit states}

We now obtain a general volume monogamy relation for $n$-qubit states, pure or mixed, based on Eq. 22 for 3qubit states. In particular, for an $n$-qubit state $\rho_{A B C D} \ldots$ consider the normalised volumes $v_{B \mid A}, v_{C \mid A}, v_{D \mid A}, \ldots$ of the steering ellipsoids generated by Alice's local measurements. The steered parties $B, C, D, \ldots$ can be grouped into $\frac{1}{2}(n-1)(n-2)$ distinct pairs, with each pair satisfying a volume monogamy relation as per Eq. (22). Summing these relations over all such pairs and rearranging terms then yields the general monogamy relation

$$
\left(v_{B \mid A}\right)^{2 / 3}+\left(v_{C \mid A}\right)^{2 / 3}+\left(v_{D \mid A}\right)^{2 / 3}+\ldots \leq \frac{n-1}{2} .
$$

This reduces to the 3 -qubit relation for $n=3$, and in general places a nontrivial constraint on the degree to which Alice can steer the states of the other parties. For example, noting that $v \leq v^{2 / 3}$ for $v \leq 1$, it follows that the average volume of the $n-1$ ellipsoids to which Alice can steer the other parties is bounded by

$$
\bar{v}_{\mid A}:=\frac{v_{B \mid A}+v_{C \mid A}+v_{D \mid A}+\ldots}{n-1} \leq \frac{1}{2} .
$$

For the 4-qubit case, the upper bound in Eq. (37) is $3 / 2$. While this is weaker than the upper bound of 1 in Eq. 28 for pure 4-qubit states, it has the advantage of also being valid for the mixed case.

\section{VOLUME MONOGAMY AND NOISE}

\section{A. Local noise and steering ellipsoids}

Taking into account the imperfections of any experiment, including in state preparation and measurement, the quantum state is inevitably exposed to all kinds of noise. Such noise processes can be modeled as a noisy channel acting on an ideal initial state. We are interested in the problem of how such channels affect the desired properties of the initial state, and in particular the steering ellipsoid.

Mathematically, a noisy channel acting on a bipartite state $\rho_{A B}$ is equivalent to a completely positive and trace preserving (CPTP) map, $\Phi$, mapping the initial state $\rho_{A B}$ to some $\rho_{A B}^{\prime}$. Here, we consider the case that the noise acts locally on each subsystem. Thus, $\Phi=\phi_{A} \otimes \phi_{B}$ where $\phi_{A}$ and $\phi_{B}$ are CPTP maps acting on $A$ and $B$, respectively, and

$$
\rho_{A B}^{\prime}=\Phi\left(\rho_{A B}\right)=\left(\phi_{A} \otimes \phi_{B}\right)\left(\rho_{A B}\right) .
$$

The set of reduced states generated by Alice's local measurements on $\rho_{A B}^{\prime}$ follows from Eq. (3) of Sec. II as

$$
\begin{aligned}
\left\{\rho_{B}^{\prime E}\right\} & =\left\{\frac{\operatorname{Tr}_{A}\left[\left(\phi_{A} \otimes \phi_{B}\right)\left(\rho_{A B}\right) E \otimes \mathbb{1}_{B}\right]}{\operatorname{Tr}\left[\left(\phi_{A} \otimes \phi_{B}\right)\left(\rho_{A B}\right) E \otimes \mathbb{1}_{B}\right]}\right\} \\
& =\left\{\frac{\operatorname{Tr}_{A}\left[\left(I_{A} \otimes \phi_{B}\right)\left(\rho_{A B}\right) \phi_{A}^{\star}(E) \otimes \mathbb{1}_{B}\right]}{\operatorname{Tr}\left[\left(I_{A} \otimes \phi_{B}\right)\left(\rho_{A B}\right) \phi_{A}^{\star}(E) \otimes \mathbb{1}_{B}\right]}\right\},
\end{aligned}
$$

where $E$ ranges over all positive operators, $I$ denotes the identity map, and the dual map $\phi^{\star}$ of any $\mathrm{CP}$ map $\phi$ is defined by $\operatorname{Tr}\left[\phi^{\star}(X) Y\right]:=\operatorname{Tr}[X \phi(Y)]$. Noting $\phi_{B}$ is trace preserving and that $\phi_{A}^{\star}$ maps positive operators to positive operators then yields

$$
\begin{aligned}
\left\{\rho_{B}^{\prime E}\right\} & =\left\{\frac{\phi_{B}\left(\operatorname{Tr}_{A}\left[\rho_{A B} \phi_{A}^{\star}(E) \otimes \mathbb{1}_{B}\right]\right)}{\operatorname{Tr}\left[\rho_{A B} \phi_{A}^{\star}(E) \otimes \mathbb{1}_{B}\right]}\right\} \\
& \subseteq\left\{\frac{\phi_{B}\left(\operatorname{Tr}_{A}\left[\rho_{A B} E \otimes \mathbb{1}_{B}\right]\right)}{\operatorname{Tr}\left[\rho_{A B} E \otimes \mathbb{1}_{B}\right]}\right\} \\
& =\left\{\phi_{B}\left(\frac{\operatorname{Tr}_{A}\left[\rho_{A B} E \otimes \mathbb{1}_{B}\right]}{\operatorname{Tr}\left[\rho_{A B} E \otimes \mathbb{1}_{B}\right]}\right)\right\} .
\end{aligned}
$$

Hence, the steering ellipsoids of $\rho_{A B}^{\prime}$ and $\rho_{A B}$ are related by

$$
\mathcal{E}_{B \mid A}^{\prime} \subseteq \phi_{B}\left(\mathcal{E}_{B \mid A}\right)
$$


To determine how the volumes of the steering ellipsoids are related, note that the trace distance between two states contracts under any CPTP map $\phi$ [1]. Moreover, for qubits, the trace distance is proportional to the Euclidean distance in the Bloch ball [1]. As a consequence, the volume of any set of qubit Bloch vectors contracts under CPTP maps, yielding the inequality chain

$$
V\left(\mathcal{E}_{B \mid A}^{\prime}\right) \leq V\left(\phi_{B}\left(\mathcal{E}_{B \mid A}\right)\right) \leq V\left(\mathcal{E}_{B \mid A}\right)
$$

via Eq. 40. Thus, local noise never increases the volume of the steering ellipsoid.

An immediate consequence of this result is that any volume monogamy relation, for a given set of multiqubit states, will remain valid under the addition of local noise. For example, it follows via Eq. (9) that

$$
\sqrt{v_{B \mid A}^{\prime}}+\sqrt{v_{C \mid A}^{\prime}} \leq 1
$$

for any state obtained by adding local noise to a pure 3qubit state, i.e., for any 3-qubit state of the form $\rho_{A B C}^{\prime}=$ $\left(\phi_{A} \otimes \phi_{B} \otimes \phi_{C}\right)\left(\left|\psi_{A B C}\right\rangle\left\langle\psi_{A B C}\right|\right)$.

\section{B. An example: local isotropic noise}

We consider the set of states

$$
\left|\varphi_{A B C}\right\rangle=p|100\rangle+\sqrt{\frac{1-p^{2}}{2}}|010\rangle+\sqrt{\frac{1-p^{2}}{2}}|001\rangle,
$$

with $p \in(0,1)$. These states are symmetric with respect to Bob and Charlie, so that $v_{B \mid A}=v_{C \mid A}$. Moreover, it is easy to verify that these are $\mathrm{W}$-class states [23]. Thus, they saturate the volume monogamy relation in Eq. (9) (see Sec. III B), making them of experimental interest.

However, the inevitable presence of noise in any experiment means that in practice these states cannot be perfectly generated and measured. We therefore investigate the robustness of these states under a simple noise model. In particular, we consider the addition of local isotropic noise,

$$
\rho_{A B C}^{\prime}=\Phi_{\varepsilon}\left(\rho_{A B C}\right):=\left(\phi_{\varepsilon} \otimes \phi_{\varepsilon} \otimes \phi_{\varepsilon}\right)\left(\rho_{A B C}\right),
$$

where

$$
\phi_{\varepsilon}: \rho \rightarrow \frac{\varepsilon}{2} \mathbb{1}+(1-\varepsilon) \rho
$$

corresponds to adding isotropic noise of strength $\varepsilon \in$ $[0,1]$. Thus, for each qubit, $\varepsilon=0$ corresponds to no noise, while $\varepsilon=1$ corresponds to noise so strong that the state becomes completely mixed. The Bloch vector of each qubit is scaled by $1-\varepsilon$.

The noisy channel $\Phi_{\varepsilon}$ preserves the symmetry of the state $\rho_{A B C}$ with respect to Bob and Charlie, and the volumes of the steering ellipsoid may be analytically calculated for the states in Eq. (43) as

$$
v_{B \mid A}^{\prime}=v_{C \mid A}^{\prime}=\frac{4 p^{4}\left(1-p^{2}\right)^{2}(1-\varepsilon)^{6}}{\left[1-(1-\varepsilon)^{2}\left(1-2 p^{2}\right)^{2}\right]^{2}} .
$$

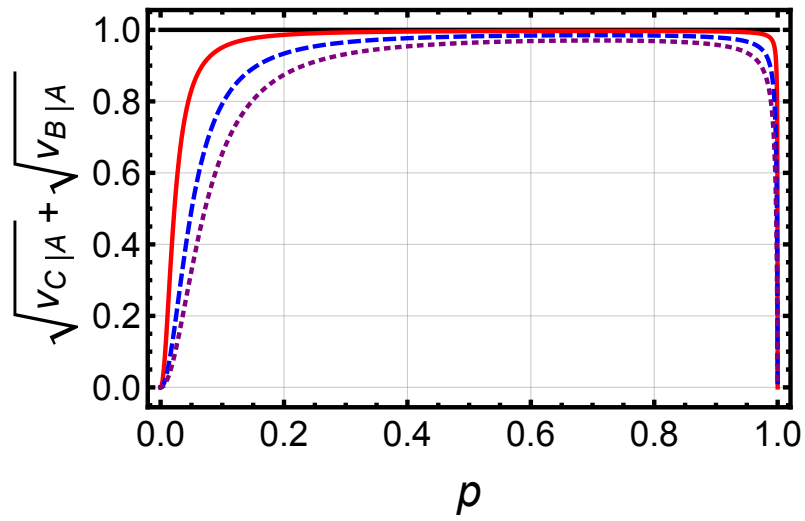

FIG. 2. The effect of local noise on the lefthand-side of the volume monogamy relation, Eq. (9), for the family of states in Eq. 43. Here, the noise on each qubit is a depolarizing channel with strength $\varepsilon=0$ (black solid curve), 0.001 (red solid curve), 0.005 (blue dashed curve), 0.01 (purple dotted curve).

The corresponding sensitivity of the volume monogamy relation (9) to noise is depicted in Fig. IV B , for a range of experimentally relevant noise strengths. It is seen that while the relation is no longer saturated for $\varepsilon>0$, those states with $p$ taking values in the midrange of the unit interval are relatively robust.

\section{CONCLUSION}

We have studied volume monogamy relations for multiqubit systems. We have demonstrated a close connection between the volume monogamy relation (9) for pure 3qubit states and the SLOCC classification of such states. A counterexample 20 was constructed to show this relation does not generalise to all 3-qubit states, and a suitable universal volume monogamy relation 22 was obtained for the general case. A similar relation was obtained in Eq. (28) for pure 4-qubit states, and conjectured to also hold for mixed states. Furthermore, we have found a generalised volume monogamy relation valid for all multi-qubit states. Finally, we studied the effects of noise on the quantum steering ellipsoid and showed that local noise channels do not invalidate the volume monogamy relation, as such noise decreases the volume of steering ellipsoids.

More generally, it is remarkable that the simple concept of the steering ellipsoid, i.e., the set of Bob's local states that Alice can prepare by local measurements on her system, can geometrically capture many important aspects of quantum correlations and information processing tasks. Properties of steering ellipsoids are not only strongly connected to quantum monogamy, as investigated here, but have also been closely linked with quantum communication protocols based on, for example, Bell nonlocality [7, teleportation [7, and Einstein-Podolsky- 
Rosen steering [8, 11].

It is hoped that further investigation of steering ellipsoids (and their generalisations to higher dimensions) will cement their relevance to understanding the properties and usefulness of quantum correlations. This goes well beyond properties of ellipsoid volumes: for example, while local dissipation reduces volumes as per Sec. IV, it is also known that such noise can enhance teleportation fidelity for some states 25. Hence, a geometric characterisation of such states will require consideration of aspects other than volume (such as semiaxis lengths [7).

Many open questions remain for future work even within the confines of volume monogamy relations. For example, can stronger monogamy relations than Eqs. (22) and (28) be obtained? Is the 4-qubit conjecture in Eq. (36) valid? Is there some underlying con- nection between volume monogamy relations and that of other types of entanglement [26 28]? How close might we get to these bounds with experiments? Finally, can volume monogamy be generalised to higher-dimensional systems?

\section{ACKNOWLEDGMENTS}

We thank Jianxin Chen for his assistance in finding the counterexample 20). S. C. thanks Li Li for helpful discussions. This work was supported by the ARC Centre of Excellence CE110001027. A. M. is supported by the EPSRC.
[1] M. A. Nielsen and I. L. Chuang, Quantum Computation and Quantum Information (Cambridge University Press, 2010).

[2] H. M. Wiseman and G. J. Milburn, Quantum Measurement and Control (Cambridge University Press, 2009).

[3] S. Jevtic, M. Pusey, D. Jennings, and T. Rudolph, Phys. Rev. Lett. 113, 020402 (2014)

[4] M. Shi, F. Jiang, C. Sun, and J. Du, New J. Phys. 13, 073016 (2011)

[5] M. Shi, C. Sun, F. Jiang, X. Yan, and J. Du, Phys. Rev. A 85, 064104 (2012)

[6] A. Milne, S. Jevtic, D. Jennings, H. Wiseman, and T. Rudolph, New J. Phys. 16, 083017 (2014)

[7] A. Milne, D. Jennings, S. Jevtic, and T. Rudolph, Phys. Rev. A 90, 024302 (2014).

[8] S. Jevtic, M. J. W. Hall, M. R. Anderson, M. Zwierz, and H. M. Wiseman, J. Opt. Soc. Am. B 32, A40 (2015)

[9] Q. Quan, H. Zhu, S.-Y. Liu, S.-M. Fei, H. Fan, and W.-L. Yang, Sci. Rep. 6, 22025 (2016).

[10] H. C. Nguyen and T. Vu, Phys. Rev. A 94, 012114 (2016)

[11] H. C. Nguyen and T. Vu, Europhys. Lett. 115, 10003 (2016)

[12] V. Coffman, J. Kundu, and W. K. Wootters, Phys. Rev. A 61, 052306 (2000)

[13] T. J. Osborne and F. Verstraete, Phys. Rev. Lett. 96, 220503 (2006)

[14] V. Scarani and N. Gisin, Phys. Rev. Lett. 87, 117901 (2001)
[15] B. Toner and F. Verstraete, arXiv: quant-ph/0611001 (2006).

[16] B. Toner, Proc. R. Soc. A 465, 59 (2009)

[17] P. Kurzyński, T. Paterek, R. Ramanathan, W. Laskowski, and D. Kaszlikowski, Phys. Rev. Lett. 106, 180402 (2011)

[18] M. D. Reid, Phys. Rev. A 88, 062108 (2013).

[19] A. Klyachko, arXiv: quant-ph/0409113 (2004).

[20] X. Hu and H. Fan, Phys. Rev. A 91, 022301 (2015).

[21] A. Milne, S. Jevtic, D. Jennings, H. Wiseman, and T. Rudolph, New J. Phys. 17, 019501 (2015)

[22] A. Higuchi, A. Sudbery, and J. Szulc, Phys. Rev. Lett. 90, 107902 (2003).

[23] W. Dür, G. Vidal, and J. I. Cirac, Phys. Rev. A 62, $062314(2000)$

[24] H.-H. Qin, S.-M. Fei, and X. Li-Jost, Phys. Rev. A 92, 062339 (2015)

[25] P. Badziag, M. Horodecki, P. Horodecki, and R. Horodecki, Phys. Rev. A 62, 012311 (2000)

[26] B. Regula, S. Di Martino, S. Lee, and G. Adesso, Phys. Rev. Lett. 113, 110501 (2014)

[27] Y.-K. Bai, Y.-F. Xu, and Z. D. Wang, Phys. Rev. Lett. 113, 100503 (2014)

[28] C. Eltschka and J. Siewert, Phys. Rev. Lett. 114, 140402 (2015). 